\title{
Mitgliederschwund und Überalterung der Parteien: Prognose der Mitgliederzahlen bis 2040
}

\author{
Nicolai Dose und Anne-Kathrin Fischer
}

Die großen Mitgliederverluste, die die deutschen Parteien seit zwei Jahrzehnten hinnehmen mussten, stellt sie vor große Schwierigkeiten. Verursacht wird der Mitgliederrückgang durch die hohe Anzahl an Austritten bei gleichzeitig relativ wenigen Eintritten ${ }^{1}$ sowie in Zukunft insbesondere durch die deutliche Zunahme an Todesfällen infolge der Überalterung des Mitgliederstamms. Mitgliederschwund ist zwar vor allem ein Problem der beiden großen Parteien SPD und CDU. Allerdings leiden auch die kleineren Parteien unter rückläufigen Mitgliederzahlen. ${ }^{2}$ Vor allem die ungünstige Altersstruktur der Parteien wird diesen in $\mathrm{Zu}$ kunft einen zum Teil dramatischen Mitgliederschwund bescheren, so das zentrale Argument dieses Beitrags, denn die starke Gruppe der heute 60-Jährigen wird in 20 Jahren in einem Alter sein, in dem der Tod nichts Ungewöhnliches ist. Zwar finden sich in der Literatur gelegentlich Hinweise auf diesen Sachverhalt ${ }^{3}$, auch tauchen hin und wieder Zahlen in der Presse auf; ausgearbeitete Prognosen der zukünftigen Mitgliederentwicklung der Parteien sind jedoch kaum zugänglich.

\section{Mitgliederschwund von Parteien: Erster Befund und Probleme}

Im Vergleich der Mitgliederstände in der Zeit kurz nach der Wiedervereinigung (Ende 1990) mit denen Ende 2012 verlor die SPD mit 49,4 Prozent fast die Hälfte ihrer Mitglieder. ${ }^{4}$ Auch die CDU musste einen gravierenden Verlust von 39,7 Prozent im betrachteten Zeitraum hinnehmen. Der Rückgang bei der CSU fiel mit 20,5 Prozent etwas gemäßigter aus. Die FDP und Die Linke übertreffen mit einem Schwund von 65,1 und 77,3 Prozent deutlich die beiden Mitgliederparteien, wobei die größten Verluste in den ersten drei Jahren nach der Wiedervereinigung zu verzeichnen waren. Bündnis 90/Die Grünen konnten hingegen als einzige im Bundestag vertretene Partei einen Zuwachs von 44,4 Prozent verbuchen.

Konzentriert man sich auf die Mitgliederentwicklung im engeren Zeitraum von 2001 bis 2012 (eigene Berechnung auf der Basis der Daten von Oskar Niedermayer 5 ) zeigt sich, dass die SPD im Laufe dieser zwölf Jahre ein Drittel ihrer Mitglieder verloren hat (33,5 Prozent). Auch die CDU musste einen gravierenden Verlust von 21,2 Prozent hinnehmen. Die Linke folgt mit einer Negativentwicklung von 18,1 Prozent in einem etwas geringeren Ausmaß.

1 Vgl. Nicolai Dose, Warum schrumpfen Mitgliederparteien?, in: Gesellschaft, Wirtschaft, Politik, 61. Jg. (2012), H. 3, S. 293 - 302.

2 Elmar Wiesendahl, Parteien, Sonderauflage für die Zentralen für politische Bildung, Frankfurt am Main 2005, S. 30.

3 Vgl. Markus Klein, Wie sind Parteien gesellschaftlich verwurzelt?, in: Tim Spier / Markus Klein I Ulrich von Alemann / Hanna Hoffmann / Anika Laux / Alexandra Nonnenmacher / Katharina Rohrbach (Hrsg.), Parteimitglieder in Deutschland, Wiesbaden 2011, S. 45.

4 Vgl. Oskar Niedermayer, Parteimitglieder in Deutschland: Version 2013, in: Arbeitshefte aus dem Otto-Stammer-Zentrum, Nr. 20 (2013), S. 2.

5 Vgl. ebenda. 


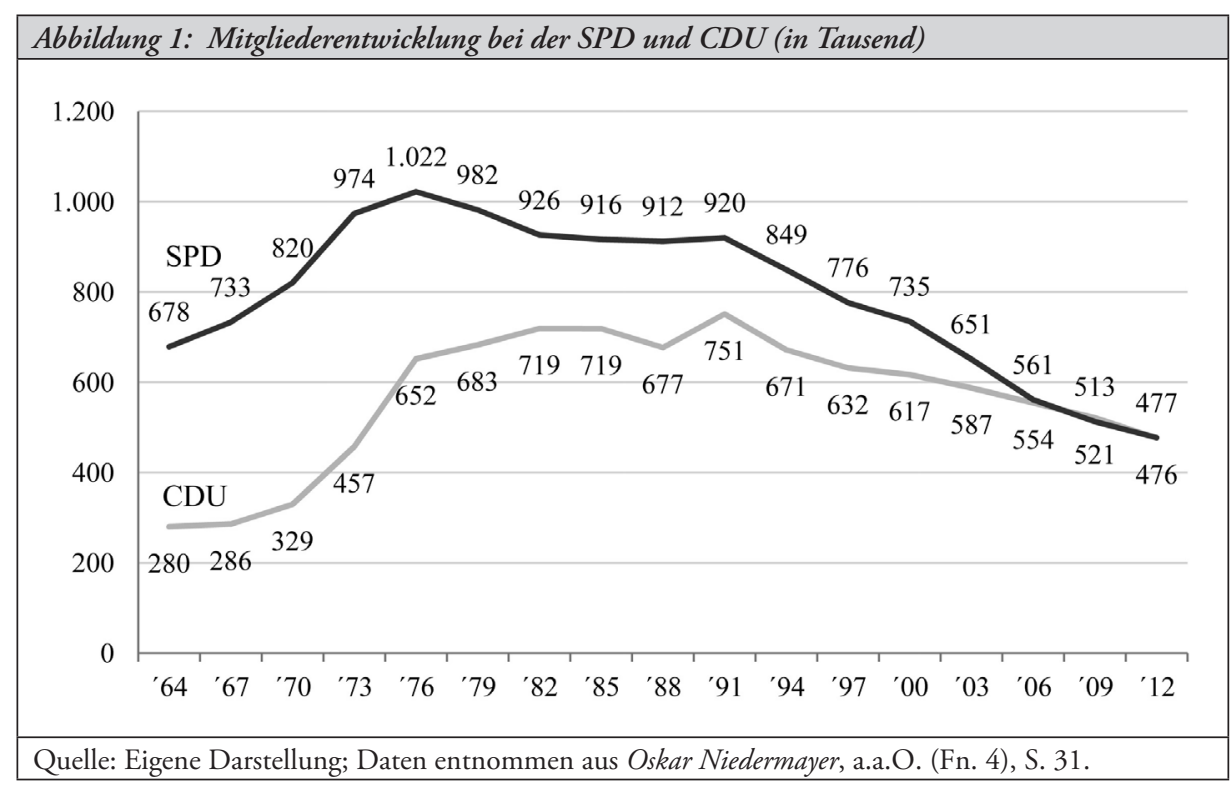

Den geringsten Mitgliederrückgang hat die FDP mit einem Minus von 8,4 Prozent zu verzeichnen. Nur die Mitgliedschaft von Bündnis 90/Die Grünen wuchs auch in diesem Zeitraum von 2001 bis 2012, und zwar um 35,4 Prozent, wobei 2010 und 2011 besonders hohe Zuwächse von jeweils über zehn Prozent gegenüber dem Vorjahr zu verzeichnen waren; 2012 reduzierte sich das Mitgliederwachstum jedoch deutlich auf 1,0 Prozent.

Betrachtet man den Mitgliederschwund bei den beiden großen Parteien, wird deutlich, dass dieser gemessen an den ehemaligen Höchstständen dramatisch ist. Die SPD wies zur Zeit ihrer höchsten Popularität (1975) deutlich über eine Million Mitglieder auf - und dies auf der Basis einer deutlich geringeren Bevölkerungszahl. Bis zum Jahre 2012 hat sich die Zahl der Mitglieder auf 477.037 mehr als halbiert. Auch die Mitgliederzahl der CDU hat sich seit dem Höchststand kurz nach der Wiedervereinigung (789.000) auf etwa den gleichen aktuellen Wert wie bei der SPD merklich verkleinert (476.347) (vgl. Abbildung 1).

Der Verlust eines Mitglieds trifft die Partei auf verschiedenen Ebenen. Parteimitglieder bilden unter anderem eine finanzielle und personelle Ressource für ihre Organisationen. Sie gelten als finanzielle Ressource, weil sie Mitgliedsbeiträge entrichten und gegebenenfalls zusätzlich spenden. Bei den beiden Großparteien machen die Mitgliedsbeiträge circa 40 Prozent (CDU) und circa 50 Prozent (SPD) der Einnahmen aus, wobei sie insbesondere in die Parteigliederungen vor Ort fließen. Aber selbst bei den Bundesparteien, die sich vor allem durch staatliche Zuschüsse finanzieren, machen die Mitgliedsbeiträge noch etwa 20 Prozent ihrer Mittel aus. Insgesamt lässt sich festhalten, dass der Anteil der Mitgliedsbeiträge an den Gesamteinnahmen der Parteien zwar zurückgegangen ist, sie aber noch immer eine wichtige Rolle spielen. Ihr Wegfall würde zu einer substantiellen Schwächung der Parteien führen. ${ }^{6}$

6 Vgl. Klaus Detterbeck, Die Relevanz der Mitglieder: Das Dilemma effektiver Partizipation, in: Uwe Jun / Oskar Niedermayer / Elmar Wiesendahl (Hrsg.), Zukunft der Mitgliederpartei, Opladen 2009, S. $71-88$, S. 79. 
Dies gilt insbesondere, weil der Umfang der staatlichen Parteienfinanzierung nach geltender Rechtslage unter anderem an das Beitrags- und Spendenaufkommen gekoppelt ist. ${ }^{7}$

Mitglieder stellen aber auch eine personelle Ressource für die Parteiarbeit dar: angefangen bei Einsätzen als Verteiler von Werbematerial, über die Wahrnehmung unterer und mittlerer Führungspositionen bis hin zu der des Bundesvorsitzenden. Ohne eine breite Mitgliederbasis wäre es nicht möglich, alle Ämter und Mandate zu besetzen. Schon jetzt gelingt es den Parteien zunehmend nicht mehr, ausreichend viele Kandidaten aufzustellen. Gerade die Partizipation einer Vielzahl von Mitgliedern ist für den Fortbestand einer Partei unerlässlich. ${ }^{8}$ Auch ermöglichen Parteimitglieder eine Integration der Parteien und ihrer politischen Ideen in die Gesellschaft; sie dienen der Repräsentation der Partei. ${ }^{9}$ Den Mitgliedern kommt eine Multiplikatorenfunktion zu: Sie nehmen Einfluss auf das persönliche Umfeld und verbreiten die politischen Ansichten der Partei im direkten Kontakt. ${ }^{10}$ Darüber hinaus gelten Mitglieder als loyale und sichere Wähler. Generell leisten Mitglieder einen Beitrag zur Akzeptanz und demokratischen Legitimität ihrer Partei. Ist die Mitgliederbasis breit ausgeprägt, hat dies Auswirkungen auf die Stärke der gesellschaftlichen Repräsentanz. Gerade eine solche Breite gehört zum Selbstbild der deutschen Mitgliederparteien, die zumindest dem Anspruch nach auch immer Volksparteien seien wollen. ${ }^{11}$ Sind gar vor Ort keine Parteistrukturen mehr vorhanden, finden Interessierte und damit potenzielle Neumitglieder auch keine lokalen Ansprechpartner mehr. Insgesamt scheint der deutliche Mitgliederverlust insbesondere die finanzielle und organisatorische Basis der beiden Mitgliederparteien SPD und CDU ins Wanken zu bringen und vor große Probleme zu stellen. ${ }^{2}$

Betrachtet man die aktuelle Mitgliederentwicklung und vor allem die Todesfälle, lassen sich auch hier bereits interessante Tendenzen aufzeigen. Im Jahr 2012 hat Die Linke 2,3 Prozent des Mitgliederbestands des Vorjahrs allein durch Tod verloren. ${ }^{13}$ Mit deutlichem Abstand folgen SPD, CDU und CSU, in deren Reihen zwischen 1,4 und 1,6 Prozent der Mitglieder innerhalb eines Jahres verstarben. Die FDP erleidet einen vergleichsweise moderaten Verlust durch Todesfälle (0,7 Prozent), Bündnis 90/Die Grünen mit 0,3 Prozent den geringsten. Diese Schwundraten waren über die letzten Jahre bei allen genannten Parteien sehr stabil.

Vor diesem Hintergrund ist eine fundierte Prognose der zukünftigen Entwicklung der Mitgliederzahlen der Parteien von einiger Bedeutung. Wie aus Abbildung 2 ersichtlich, übersteigt bei allen Parteien mit deutlich hervortretender Ausnahme von Bündnis 90/ Die Grünen und möglicherweise der Piraten schon jetzt die Zahl der zu verzeichnenden Austrit-

7 Vgl. Wilhelm Hofmann / Nicolai Dose / Dieter Wolf, Politikwissenschaft, Konstanz 2010, S. $141 \mathrm{ff}$.

8 Vgl. Serge Embacher, Demokratische Beteiligungsprozesse initiieren, solidarisches Denken und Handeln fördern: Neue Strategien für Parteien und Gewerkschaften, Bonn 2010, S. 36.

9 Vgl. Heiko Biehl, Parteimitglieder im Wandel: Partizipation und Repräsentation, Wiesbaden 2005, S. 36 ff.; Elmar Wiesendahl, Die Mitgliederparteien zwischen Unmodernität und wieder entdecktem Nutzen, in: Uwe Jun / Oskar Niedermayer / ders. (Hrsg.), Zukunft der Mitgliederpartei, Opladen 2009, S. 33 - 51, S. 44; Klaus Detterbeck, Parteien und Parteiensystem, Konstanz 2011, S. 135 ff.

10 Vgl. Klaus Detterbeck, a.a.O. (Fn. 6), S. 80.

11 Vgl. ebenda; ergänzend Andreas Kießling, Politische Kultur und Parteien in Deutschland: Sind die Parteien reformierbar?, in: APuZ, B 10 (2001), S. 29 - 37, S. 32.

12 Vgl. Karl-Rudolf Korte, So entscheiden Parteien: Umfeld-Bedingungen innerparteilicher Partizipation, in: ders. I Jan Treibel (Hrsg.), Wie entscheiden Parteien? Prozesse innerparteilicher Willensbildung in Deutschland, ZPol-Sonderband, Baden-Baden 2012, S. 267 - 286, S. 276.

13 Vgl. Oskar Niedermayer, a.a.O. (Fn. 4), S. 25. 


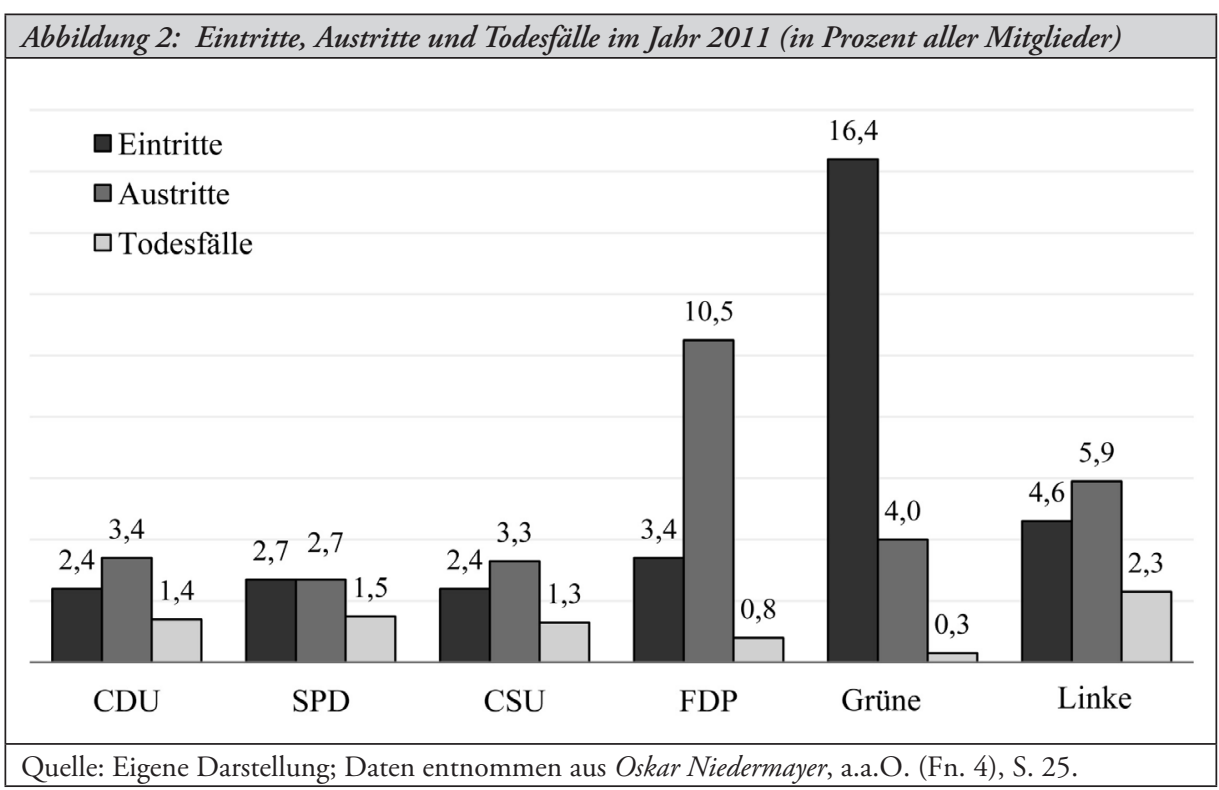

te die Zahl der Eintritte. Bei der SPD halten sich diese bei einer prozentualen Betrachtung die Waage; allerdings zeigen die absoluten Zahlen, dass die Austritte noch die Eintritte überwiegen. Dabei könnte die Zahl der Austritte sogar noch unterschätzt werden, weil in den zur Verfügung stehenden Statistiken lediglich die expliziten Austritte erfasst werden. Der tatsächliche Verlust an Mitgliedern, der auch durch Wegzug, Karteibereinigung, nicht mehr klärbarem Abgang, Ausschluss oder Ausscheiden wegen Beitragsrückstands zustande kommt, wird nicht voll erfasst.

Rechnet man die Todesfälle hinzu, die für sich genommen momentan bei allen Parteien noch durch die Eintritte kompensiert werden können, ergibt sich ein wenig erfreuliches Bild. ${ }^{14}$ In Zukunft wird sich ihre Bedeutung wegen der Überalterung vieler Parteien verstärken. Deshalb konzentrieren wir uns an dieser Stelle auf den Mitgliederverlust, auf den die Parteien sich allein aufgrund von Sterbefällen einstellen müssen. Der Schwund, der sich zusätzlich aus dem Saldo von Eintritten und Austritten ergibt, kann nicht in die Berechnung einbezogen werden, da sich diese Entwicklung derzeit nicht über einen größeren Zeitraum seriös abschätzen lässt. Allerdings führte der Saldo aus Eintritten und Austritten bisher zu einer zusätzlichen Verringerung der Mitgliederzahlen. Wenn also aus methodischen Gründen für die nachfolgende Berechnung angenommen wird, dass es zu keinerlei Eintritten und Austritten kommen wird, werden die zukünftigen Probleme für die Parteien noch unterschätzt. Um die Prognose der zukünftigen Mitgliederzahlen unter der getroffenen Annahme vornehmen zu können, wird der derzeitige Altersaufbau der Parteimitgliedschaften mit den aktuellen Generationensterbetafeln in Beziehung gesetzt. Da der Altersaufbau bekannt ist, lässt sich auf diese Weise die zukünftige Mitgliederentwicklung, wie sie sich aufgrund von Sterbefällen ergibt, recht gut abschätzen. 


\section{Datenbasis und Ansatz der Prognose}

Für die anzustellende Prognose wurde auf die von Oskar Niedermayer dokumentierten Mitgliederzahlen (Stand 2009) zurückgegriffen. ${ }^{15}$ Der von ihm vorgelegten Parteienstatistik wurde getrennt nach Parteien die absolute Mitgliederzahl, deren Verteilung auf die einzelnen Altersgruppen - jeweils in Fünfahresschritten beginnend mit der Gruppe der 16- bis 20-Jährigen - sowie die Aufteilung der Mitglieder auf die Geschlechter entnommen. Für die Berechnung wurde, bedingt durch die Datenbasis, vereinfachend angenommen, dass die Geschlechterverteilung innerhalb der einzelnen Parteien über alle Altersklassen gleich ausfällt. Es ist jedoch davon auszugehen, dass der Anteil der weiblichen Mitglieder umso größer ausfällt, je jünger die Mitglieder der betrachteten Altersklasse sind. ${ }^{16}$ Bei der kurzfristigen Prognose werden aufgrund der Vereinfachung, dass die Verteilung zwischen Männern und Frauen für alle Altersgruppen gleich ist, die Todesfälle unterschätzt. Denn in den höheren Altersgruppen, in denen die Sterbewahrscheinlichkeit am höchsten ist, befinden sich tatsächlich deutlich mehr Männer, die eine höhere Sterbewahrscheinlichkeit aufweisen, als in der Rechnung angenommen wird. Bei einer längerfristigen Prognose spielt diese Generation allerdings eine untergeordnete Rolle, da bei der jüngeren Generation umgekehrt eine Überschätzung der Todesfälle durch die vereinfachende Verteilung der Geschlechter auf die Altersgruppen vorliegt. In diesen Altersgruppen gibt es nämlich mehr Frauen, als in der Berechnung angenommen wird, was zu einer Überschätzung der Todesfälle pro Jahr führt. Allerdings erscheinen die Auswirkungen dieser Ungenauigkeit eher gering zu sein, da anzunehmen ist, dass sich die beiden Effekte annähernd ausgleichen.

Im weiteren Verlauf der Hochrechnung wurde aufgrund der Datenlage davon ausgegangen, dass das Alter der Mitglieder innerhalb der erhobenen, fünf Jahre umfassenden Altersklassen zwischen 16 und 85 jeweils gleich verteilt sei. Sind beispielsweise 2009 nach Parteienstatistik 16.950 weibliche SPD-Mitglieder im Alter von 51 bis 55 Jahren erfasst worden, wurde also angenommen, es wären jeweils 3.390 Frauen Mitglied der SPD, die 51, 52, 53, 54 und 55 Jahre alt sind. Obwohl diese Annahme eine erhebliche Vereinfachung darstellt, bewirkt sie keine systematische Unter- oder Überschätzung der Todesfälle und die mit ihr verbundenen Ungenauigkeiten sind mutmaßlich geringfügig und somit vernachlässigbar. Für die in der Parteienstatistik lediglich als „Über 86-Jährige“ angegebene Altersgruppe wurde in der vorliegenden Rechnung angenommen, die Mitgliederanzahl reduziere sich arithmetischdegressiv bis zum unterstellten Höchstalter für Parteimitglieder von 100 Jahren. Vor dem Hintergrund, dass die Zahl der Mitglieder, die älter als 100 Jahre sind, tatsächlich sehr niedrig ist, erscheint das gewählte Höchstalter angemessen. Der angenommene arithmetisch-degressive Verlauf der Altersstruktur verweist darauf, dass die Anzahl der Todesfälle unter den Parteimitgliedern je Jahrgang linear sinkt und dass bis zur Vollendung des 101. Lebensjahres alle Parteimitglieder versterben. Obwohl dies eine gewisse Vereinfachung darstellt, erscheint sie für die durchgeführte Berechnung als angemessen, da erstens lediglich eine sehr geringe Anzahl von Mitgliedern in dieser Altersklasse vorhanden ist, gerade weil die Sterbewahrscheinlichkeit relativ groß ist; und zweitens, weil Mitglieder in diesem hohen Alter vermehrt aus gesundheitlichen oder finanziellen Gründen austreten, wie aus einer Befragung ausgetre-

15 Vgl. Oskar Niedermayer, Parteimitgliedschaften im Jahre 2010, in: ZParl, 42. Jg. (2011), H. 2, S. $365-383$.

16 Vgl. ebenda, S. 368. 
tener Mitglieder zu erfahren war. ${ }^{17}$ Die gewonnene Altersverteilung getrennt nach Geschlechtern stellt die Basis der Hochrechnung dar und wurde für die im 17. Bundestag vertretenen Parteien CDU, SPD, CSU, Bündnis 90/Die Grünen, FDP und Die Linke erstellt.

Um den Mitgliederschwund durch Todesfälle zu berechnen, wurde des Weiteren auf die Generationensterbetafeln des Statistischen Bundesamtes ${ }^{18}$ zurückgegriffen. Sie weisen die Sterbewahrscheinlichkeit und die Lebenserwartung der jeweiligen Geburtsjahrgänge aus und erfassen auch die spezifischen Merkmale des Jahrgangs. Durch Generationssterbetafeln kann bestimmt werden, wie alt Personen im Durchschnitt werden, und sie ermöglichen Aussagen über die zukünftige Entwicklung. Die Daten des Statistischen Bundesamtes beziehen sich zwar auf Westdeutschland, können jedoch aufgrund der großen Ähnlichkeit in der Lebenserwartung und der konsistenten Entwicklung der Geburtsjahrgänge ohne größere Probleme auf das gesamte Bundesgebiet übertragen werden. ${ }^{19}$

Durch die Verwendung der bundesdurchschnittlichen Sterbetafeln wird der Einfluss von anderen, neben dem Alter auf die Sterblichkeit wirkenden soziodemographischen Parametern vernachlässigt. Tatsächlich dürfte es sozialstrukturelle Unterschiede zwischen Parteimitgliedern und der Gesamtbevölkerung geben. Vermutlich sind diese jedoch nicht so ausgeprägt, dass sie das Ergebnis der Hochrechnung signifikant beeinflussen. Es werden ausschließlich die aktuellen Mitglieder betrachtet, und es wird davon ausgegangen, dass diese bis zum Tod ihrer Partei treu bleiben. Es wird also angenommen, dass weder Parteieintritte noch -austritte erfolgen. Diese Vorgehensweise ist erforderlich, weil Parteieintritte und -austritte nicht für einen längeren Zeitraum seriös prognostiziert werden können.

Konkret wurde auf die Modellrechnungen des Statistischen Bundesamtes basierend auf einer Trendvariante für die Jahrgänge von 1910 bis 1993 zurückgegriffen, um die Sterbewahrscheinlichkeiten auf die derzeitigen Geburtenjahrgänge der Parteien anwenden zu können. Auf Basis der Mitgliederzahlen und der Generationensterbetafeln wurde entsprechend der folgenden Formel eine Hochrechnung vorgenommen:

$$
\mathrm{X}_{\mathrm{t}+1, \mathrm{a}+1}=\mathrm{X}_{\mathrm{t}, \mathrm{a}} *\left(1-\mathrm{p}_{\mathrm{a}}\right)
$$

Mit: $\mathrm{X}_{\mathrm{t}+1, \mathrm{a}+1}=$ Anzahl der Mitglieder im Jahr +1 im Alter von $\mathrm{a}+1$

$\mathrm{X}_{\mathrm{t}, \mathrm{a}}=$ Anzahl der Mitglieder im Jahr $\mathrm{t}$ im Alter von $\mathrm{a}$

$\mathrm{p}_{\mathrm{a}} \quad=$ Sterbewahrscheinlichkeit eines Mitgliedes innerhalb des a - ten Lebensjahres

Gerechnet an einem Beispiel ergibt sich für die 2.981 männlichen SPD-Mitglieder im Alter von 36 des Jahres 2009, dass 2010 noch 2.980 von ihnen am Leben und 37 Jahre alt sind:

$$
2979,6287=2.981 *(1-0,00046)
$$

Für die späteren Jahre wird entsprechend vorgegangen. Die Anzahl der Mitglieder im Alter von a +1 ergibt sich wieder aus der Anzahl der Mitglieder im Alter von a des Vorjahres multipliziert mit der Sterbewahrscheinlichkeit des entsprechenden Jahrgangs im Betrachtungsjahr.

17 Vgl. Nicolai Dose / Nathalie Golla / Anne-Kathrin Fischer, Mitgliederschwund bei der SPD: Entwicklungen und Erklärungen. Das Beispiel des Unterbezirks Siegen-Wittgenstein (Kurzfassung), Duisburg 2012.

18 Vgl. Statistisches Bundesamt, Generationensterbetafeln für Deutschland: Modellrechnungen für die Geburtsjahrgänge 1896-2009, Wiesbaden 2006.

19 Vgl. dass., Generationensterbetafeln für Deutschland: Modellrechnungen für die Geburtsjahrgänge 1871-2004, Wiesbaden 2006, S. 3. 


\section{Ergebnisse}

Die Hochrechnung macht deutlich, dass alle Parteien für die Zukunft mit einem Mitgliederverlust allein aufgrund der Sterbefälle rechnen müssen. Wie Abbildung 3 veranschaulicht, werden - wie vermutet - insbesondere die Mitgliederparteien SPD und CDU einen massiven Mitgliederschwund zu verzeichnen haben. Im Jahre 2040 werden sie jeweils noch über circa 200.000 Mitglieder verfügen. Die sehr ähnliche Altersstruktur von CDU und SPD sorgt für nahezu parallel verlaufende Schrumpfungsprozesse.

Die Mitgliederzahlen von Bündnis 90/Die Grünen werden hingegen vergleichsweise stabil bleiben. Die CSU wird bis 2040 nach einem Verlust von circa 39 Prozent aufgrund von Todesfällen im Vergleich zum Ausgangsjahr 2009 noch knapp 62.000 Mitglieder aufweisen können - angenommen es gäbe keine Eintritte oder Austritte. Die Verluste der Liberalen und der Linken verlaufen - gemessen in absoluten Zahlen - recht ähnlich, und bereits auf den ersten Blick sieht die Entwicklung nicht so dramatisch aus wie bei den beiden Volksparteien. Um einen besseren Eindruck von den Verlusten durch Sterbefälle zu erhalten, werden in Abbildung 4 die prozentualen Verluste durch Todesfälle zum Ausgangsjahr 2009 dargestellt.

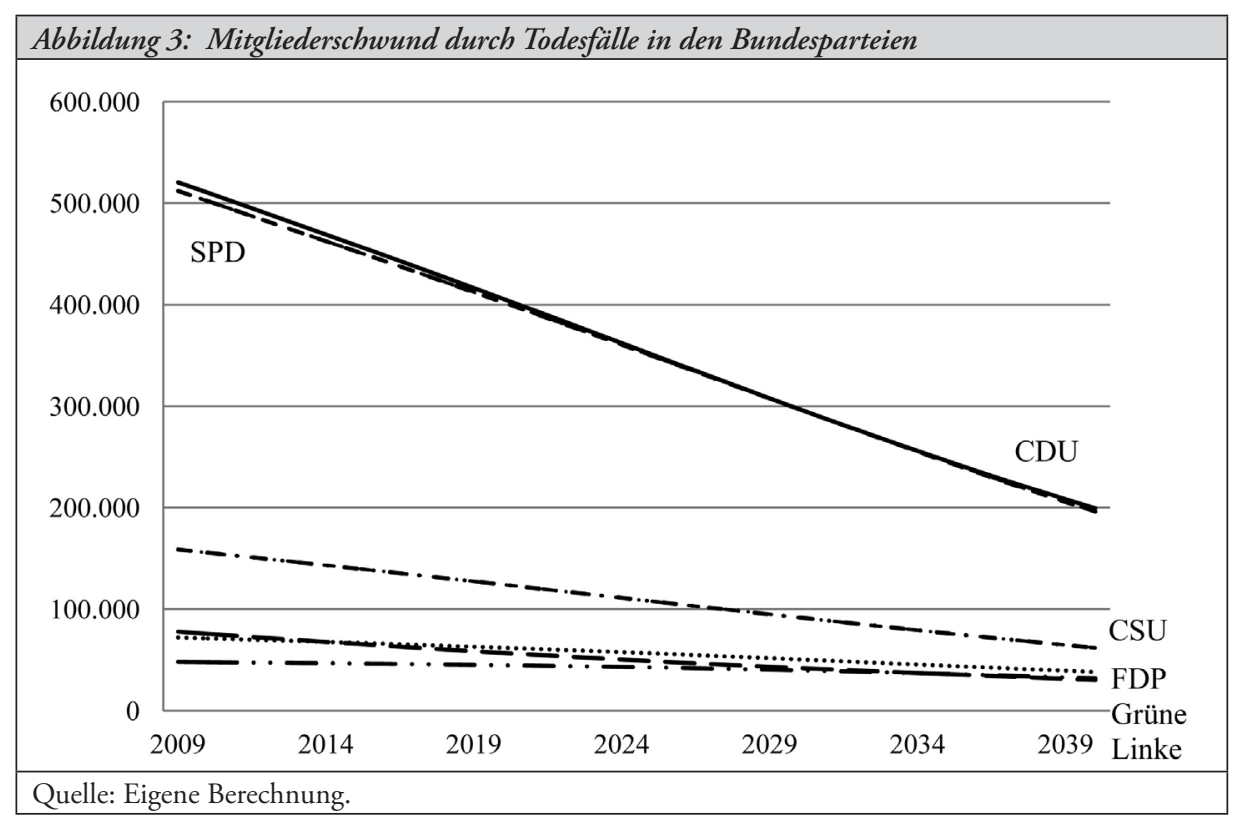

Die Linke hat prozentual den vergleichsweise stärksten Mitgliederverlust durch Todesfälle zu verzeichnen. Dies ist wenig überraschend, weil sie mit einem Durchschnittsalter von 60 Jahren die Partei mit den ältesten Mitgliedern ist. ${ }^{20}$ Der deutliche Abfall der Kurve schwächt sich jedoch im Zeitverlauf etwas ab. Für die Grünen ergibt sich nach der Hochrechnung ein beinahe umgekehrter Kurvenverlauf. Der prozentuale Anteil der Sterbefälle ist - verglichen mit dem der anderen Parteien - stets am geringsten, auch wenn im Laufe der Zeit der Verlust

20 Vgl. Oskar Niedermayer, Parteimitglieder in Deutschland: Version 2012, in: Arbeitshefte aus dem Otto-Stammer-Zentrum, Nr. 19 (2012), S. 20. 


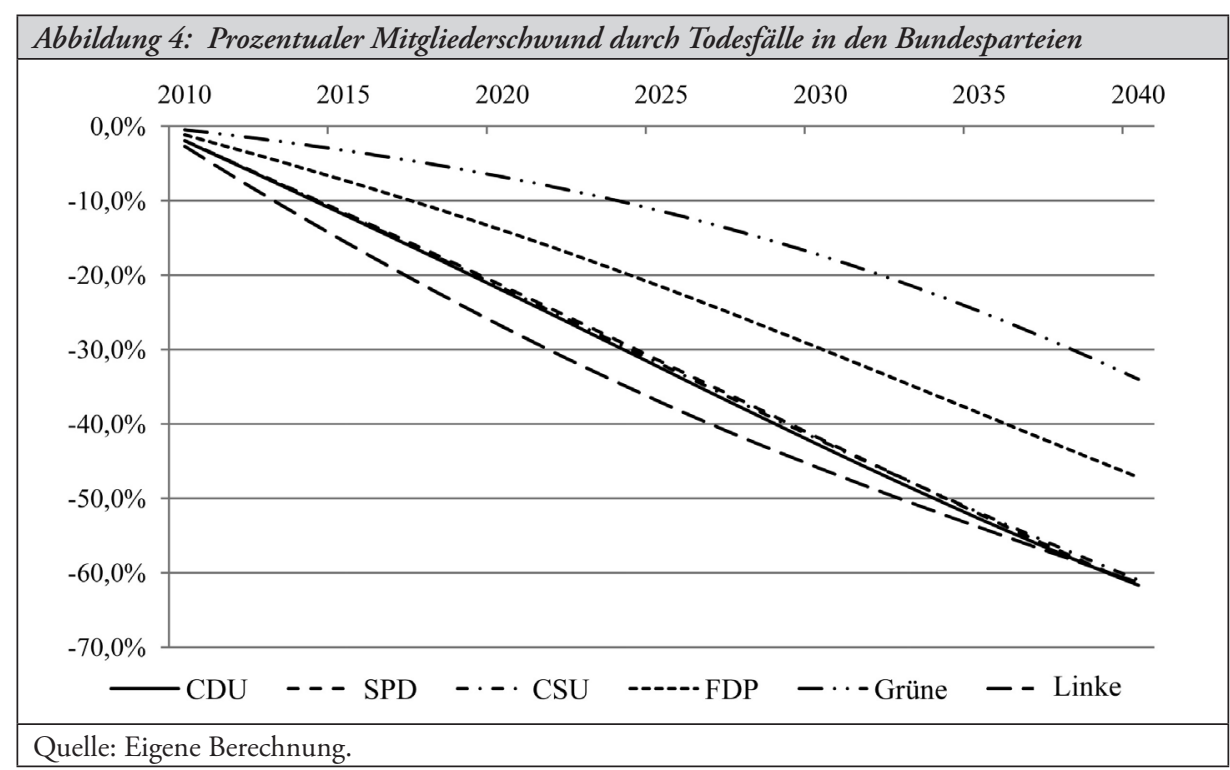

etwas größer wird. Im Gegensatz zum Durchschnittsalter der Mitglieder von der Linken (60 Jahre) weist ein Durchschnittsmitglied der Grünen ein Alter von 47 Jahren auf und ist auch deutlich jünger als die durchschnittlichen Mitglieder der anderen untersuchten Parteien. ${ }^{21}$

Der Trendverlauf der Sterblichkeitsrate ist bei den beiden Volksparteien fast linear. Auch die CSU wird prozentual in gleichem Ausmaß Mitglieder verlieren. Dieser annähernd identische Verlauf der Mitgliederparteien und der CSU verweist auf die sehr ähnliche Altersstruktur der Parteien - schließlich handelt es sich bei der CSU um die bayerische Schwesterpartei der CDU. Diese sehr ähnliche Altersstruktur zeigt sich bereits an dem identischen Durchschnittsalter von 59 Jahren in den Mitgliederparteien SPD und CDU und einem ähnlichen Durchschnittsalter von 57 Jahren bei der CSU.22

Der Mitgliederschwund ist lediglich eine Ausprägung des Wandels der Parteienlandschaft. Mit der relativ geringen Zahl an Neumitgliedern und den teilweise deutlich wahrnehmbaren Austrittswellen bei einigen Parteien geht eine Lockerung der traditionellen Parteibindungen einher. Außerdem handelt es sich bei den jetzt Eintretenden um Mitglieder „neuen Typs“ die mehrheitlich nicht mehr aus den traditionellen Trägermilieus stammen und die Mitgliedschaft weniger aus Nähe zur und Identifikation mit der Politik der Partei anstreben, sondern eher aufgrund einer rationalen Kosten-Nutzen-Überlegung. ${ }^{23}$ Da gerade bei älteren und langjährigen Parteimitgliedern eine höhere Parteibindung vorliegt ${ }^{24}$, ist es umso gravierender, wenn diese durch ihr Ableben den Parteien verloren gehen und Mitglieder an ihre Stelle treten, die eine geringere Parteibindung aufweisen. So verliert eine Partei

21 Vgl. ebenda, S. 20.

22 Vgl. ebenda.

23 Vgl. Heiko Biehl, a.a.O. (Fn. 9), S. 13.

24 Vgl. Katharina Rohrbach, Warum treten Mitglieder aus?, in: Tim Spier / Markus Klein / Ulrich von Alemann / Hanna Hoffmann / Anika Laux / Alexandra Nonnenmacher / Katharina Rohrbach (Hrsg.), a.a.O. (Fn. 3), S. 177 - 202, S. 180 f. 
im Laufe der Zeit die für sie besonders wertvollen Mitglieder, die zwar zahlenmäßig zumindest zum Teil durch Neumitglieder ersetzt werden können. Diese dürften sich aber wegen ihrer geringeren Parteibindung weniger aktiv einbringen. Zudem spricht die Altersstruktur der neu eingetretenen Mitglieder nicht unbedingt für eine Verjüngung der Parteien. Das Durchschnittalter unter den Neueintritten ähnelt sich bei allen Parteien stark und liegt im Schnitt bei 41 Jahren. ${ }^{25}$

\section{Drastische Verluste an Mitgliedern und Ressourcen}

Auf Basis aktueller Generationensterbetafeln des Statistischen Bundesamtes und des bekannten Altersaufbaus der Parteien kann für die CDU, CSU, SPD und Die Linke ein Mitgliederverlust bis zum Jahre 2040 von über 60 Prozent gegenüber dem Basisjahr 2010 vorhergesagt werden. Diese Prognose beruht allein auf einer Hochrechnung der Abgänge durch Todesfälle. Die Bilanz der Ein- und Austritte konnte nicht eingerechnet werden, weil sich diese nicht seriös abschätzen lässt. Allerdings waren in den letzten Jahren bei den meisten Parteien mehr Aus- als Eintritte zu verzeichnen. Gerade die beiden Mitgliederparteien CDU und SPD haben massive Mitgliederverluste zu verzeichnen. Die CDU kam in ihrer besten Zeit Anfang der 1990er auf circa 789.000 Mitglieder, die SPD hatte in ihrer Hochzeit Mitte der 1970er Jahre gar über eine Million Mitglieder. Beide Parteien lagen im Jahre 2011 mit circa 490.000 Mitgliedern nahezu gleichauf. Bei einem Rückgang um 60 Prozent bis 2040 wiesen SPD und CDU nur noch jeweils circa 196.000 Mitglieder auf. Der Mitgliederstamm der CSU wird sich bis zum Jahr 2040 angesichts der Schrumpfungsrate von knapp über 60 Prozent auf circa 62.000 reduzieren. Für Die Linke verweist der prognostizierte Mitgliederverlust von circa 61 Prozent auf einen Mitgliederstamm von ungefähr 30.000 im Jahre 2040. Die FDP und Bündnis 90/Die Grünen werden eine weniger dramatische Schrumpfung verkraften müssen. Für die FDP läge der Verlust an Mitgliedern bis 2040 bei über 45 Prozent und bei Bündnis 90/Die Grünen bei fast 35 Prozent. Für alle Parteien, aber insbesondere für CDU, CSU, SPD und Die Linke würde der substantielle Verlust an Mitgliedern eine deutliche Reduktion der ihnen zur Verfügung stehenden personellen und finanziellen Ressourcen bedeuten. Insbesondere die Mitgliederparteien sind für Ihre Wahlkämpfe auf die aktive Mitarbeit ihrer Mitglieder angewiesen, wenn es beispielsweise darum geht, Werbematerial zu verteilen oder mit Informationsständen in der Fußgängerzone Präsenz zu zeigen. Auch müssen ausreichend viele Mitglieder als Kandidaten zu Wahlen aufgestellt werden können, was bereits beim heutigen Mitgliederbestand zunehmend weniger gelingt. Niedrige Mitgliederzahlen unterminieren darüber hinaus die demokratische Legitimität parteipolitischer Entscheidungen. Ganz unabhängig von der Beurteilung der entsprechenden Kritik dürfte beispielsweise ein Mitgliederentscheid über einen Koalitionsvertrag bei deutlich geschrumpften Mitgliederzahlen auf relativ große Vorbehalte stoßen. Schließlich bedeuten niedrigere Mitgliederzahlen niedrigere Einnahmen aus Mitgliedsbeiträgen und aus der zum Teil an die Höhe der Mitgliedsbeiträge gekoppelten staatlichen Parteienfinanzierung. Weniger hart wird es Die Grünen treffen, weil bei ihnen die Anzahl der Eintritte die der Austritte übersteigt. Alle anderen Parteien werden sich auf die neuen Gegebenheiten einstellen müssen.

25 Eigene Berechnung auf der Basis von Oskar Niedermayer, a.a.O. (Fn. 20), S. 25. 